\title{
Combined shape and topology optimization of 3D structures
}

Christiansen, Asger Nyman; Bærentzen, Jakob Andreas; Nobel-Jørgensen, Morten; Aage, Niels; Sigmund, Ole

Published in:

Computers \& Graphics

Link to article, DOI:

10.1016/j.cag.2014.09.021

Publication date:

2015

Document Version

Peer reviewed version

Link back to DTU Orbit

Citation $(A P A)$ :

Christiansen, A. N., Bærentzen, J. A., Nobel-Jørgensen, M., Aage, N., \& Sigmund, O. (2015). Combined shape and topology optimization of 3D structures. Computers \& Graphics, 46, 25-35.

https://doi.org/10.1016/j.cag.2014.09.021

\section{General rights}

Copyright and moral rights for the publications made accessible in the public portal are retained by the authors and/or other copyright owners and it is a condition of accessing publications that users recognise and abide by the legal requirements associated with these rights.

- Users may download and print one copy of any publication from the public portal for the purpose of private study or research.

- You may not further distribute the material or use it for any profit-making activity or commercial gain

- You may freely distribute the URL identifying the publication in the public portal

If you believe that this document breaches copyright please contact us providing details, and we will remove access to the work immediately and investigate your claim 


\section{Author's Accepted Manuscript}

Combined shape and topology optimization of 3D structures

Asger N. Christiansen, J. Andreas Bærentzen, Morten Nobel-Jørgensen, Niels Aage, Ole Sigmund

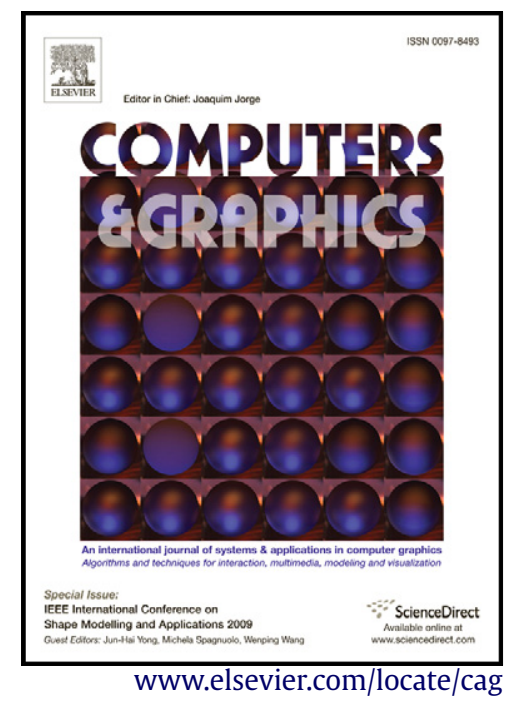

PII: S0097-8493(14)00109-5

DOI: $\quad$ http://dx.doi.org/10.1016/j.cag.2014.09.021

Reference: CAG2511

To appear in: Computers \& Graphics

Received date: 29 June 2014

Revised date: 24 August 2014

Accepted date: 16 September 2014

Cite this article as: Asger N. Christiansen, J. Andreas Bærentzen, Morten Nobel-Jørgensen, Niels Aage, Ole Sigmund, Combined shape and topology optimization of 3D structures, Computers \& Graphics, http://dx.doi.org/10.1016/j. cag.2014.09.021

This is a PDF file of an unedited manuscript that has been accepted for publication. As a service to our customers we are providing this early version of the manuscript. The manuscript will undergo copyediting, typesetting, and review of the resulting galley proof before it is published in its final citable form. Please note that during the production process errors may be discovered which could affect the content, and all legal disclaimers that apply to the journal pertain. 


\title{
Combined Shape and Topology Optimization of 3D Structures
}

\author{
Asger N. Christiansen, J. Andreas Bærentzen, Morten Nobel-Jørgensen, Niels Aage, Ole Sigmund
}

Technical University of Denmark, Denmark

\begin{abstract}
We present a method for automatic generation of 3D models based on shape and topology optimization. The optimization procedure, or model generation process, is initialized by a set of boundary conditions, an objective function, constraints and an initial structure. Using this input, the method will automatically deform and change the topology of the initial structure such that the objective function is optimized subject to the specified constraints and boundary conditions. For example, this tool can be used to improve the stiffness of a structure before printing, reduce the amount of material needed to construct a bridge, or to design functional chairs, tables, etc. which at the same time are visually pleasing.

The structure is represented explicitly by a simplicial complex and deformed by moving surface vertices and relabeling tetrahedra. To ensure a well-formed tetrahedral mesh during these deformations, the Deformable Simplicial Complex method is used. The deformations are based on optimizing the objective, which in this paper will be maximizing stiffness. Furthermore, the optimization procedure will be subject to constraints such as a limit on the amount of material and the difference from the original shape.
\end{abstract}

Keywords: Topology optimization, shape optimization, Deformable Simplicial Complex method, structural design

\section{Introduction}

2 Topology optimization is the discipline of finding the s optimal shape and topology of a structure [1][2]. It ${ }_{4}$ can be used to solve a wide variety of design problems 5 arising when producing such diverse products as cars, 6 houses, computer chips and antennas. The manufactur7 ers are often concerned with finding the stiffest struc8 ture, the lightest structure which does not break, the 9 structure with the highest cooling effect, or the structure 10 with the best flow or highest efficiency.

${ }_{11}$ With the advances in 3D printing technology, topol12 ogy optimization is not just of interest to manufactur${ }_{13}$ ers, but to anyone who has access to a $3 \mathrm{D}$ printer. ${ }_{14}$ Most consumers lack formal training in structural me${ }_{15}$ chanics, which can hinder the process with many itera${ }_{16}$ tions and costly failed attempts. Consumers can under17 engineer a design unsuitable for the intended load, or 18 over-engineer a design that wastes expensive construc19 tion material. Topology optimization offers consumers a ${ }_{20}$ tool for designing shapes that meet their structural needs ${ }_{21}$ while using minimal construction resources.

22 In this paper, we present a fully automated design tool ${ }_{23}$ for designing structurally sound structures which can be 24 manufactured, constructed or printed. The modeler only
25 has to specify boundary conditions, the optimization ${ }_{26}$ objective, constraints and an initial structure. In other ${ }_{27}$ words, the designer specifies a set of requirements (the 28 functionality of the structure and not the structure itself) ${ }_{29}$ and the method automatically designs a structure which ${ }_{30}$ fits those requirements. Note that this design process is ${ }_{31}$ significantly different from today where a designer man32 ually models a structure and requirements are taken into ${ }_{33}$ account during this design process.

${ }_{34}$ The proposed method for topology optimization is ${ }_{35}$ based on the Deformable Simplicial Complex (DSC) ${ }_{36}$ method [3]. The DSC method represents a solid struc${ }_{37}$ ture with a conforming tetrahedral mesh (a simplicial 38 complex) whose tetrahedral elements either lie entirely ${ }_{39}$ inside or outside the structure. The interface between ${ }_{40}$ solid and void (the surface) is represented explicitly by ${ }_{41}$ the triangular faces shared by an interior and exterior ${ }_{42}$ tetrahedral element. Furthermore, the DSC method en${ }_{43}$ sures well-formed tetrahedral elements by constantly ${ }_{44}$ performing mesh improvement routines while the sur${ }_{45}$ face is being deformed. Finally, it provides adaptive res${ }_{46}$ olution, allowing fine details where and when needed.

47 The method uses two optimization strategies:

\section{${ }_{48}$ Discrete optimization}




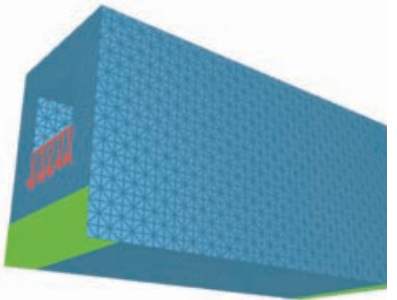

(a) Time step 0

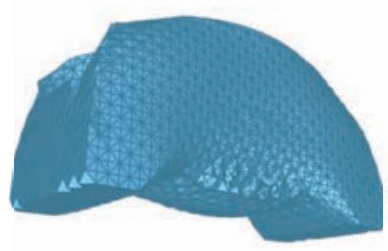

(b) Time step 10

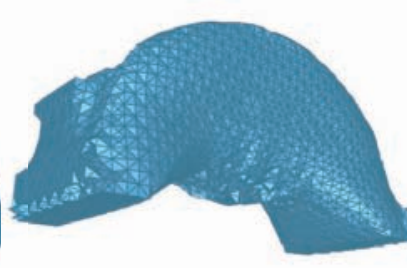

(c) Time step 20

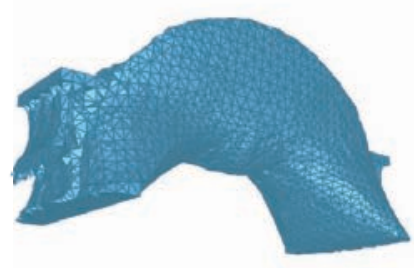

(d) Time step 30

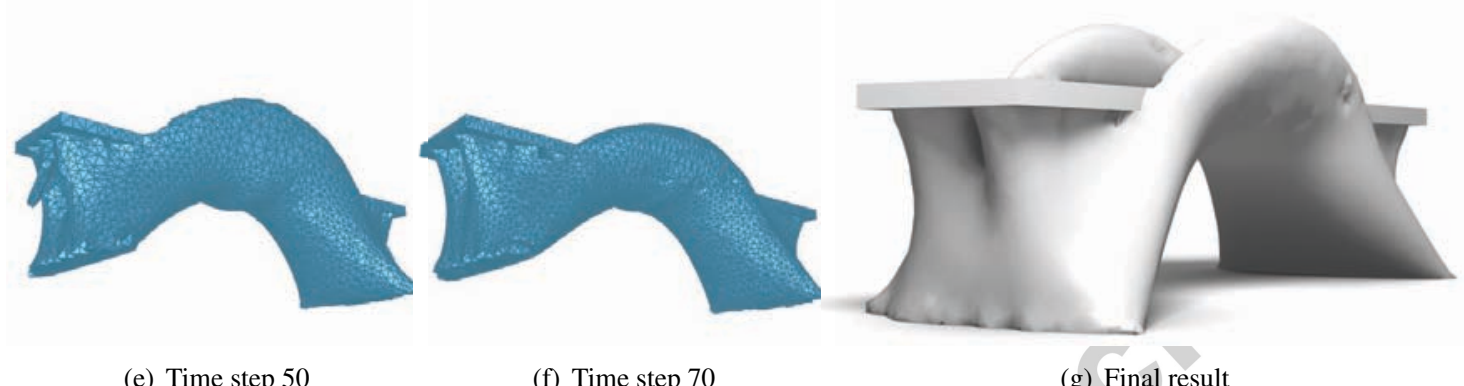

(e) Time step 50

(f) Time step 70

(g) Final result

Figure 1: Given a few input parameters, the proposed method automatically optimizes the shape and topology of a 3D structure. Here is an example of optimizing a bridge. The initial structure is seen to the upper left along with supports (green) and loads (red). This structure is optimized such that stiffness is maximized and the amount of material is minimized. A few iterations of the method are depicted along with the result.

\section{${ }_{54}$ Continuous optimization}

${ }_{65}$ These optimization strategies are iterated until changes ${ }_{66}$ are small. An example is seen in Figure 1.

${ }_{67}$ We will show that this tool is of interest to both engi68 neers and designers. For example, we show that it can 69 be used to improve stiffness and balance of a 3D model, 70 to save material and to generate functional as well as, in 71 our opinion, visually pleasing designs.

\subsection{Related work}

73. Recent trends in the computer graphics society are to 74 add mechanical properties to 3D models. Prévost et al. 75 have been concerned with the balance of printed models 76 [15], Skouras et al. about printing deformable characters 77 using a stiff and soft material [16] and several research 78 teams have focused on self-supporting masonry struc79 tures [17][18][19].

80 A major concern has been to improve the stiffness of ${ }_{81}$ 3D models. Umetani et al. perform a cross-sectional 82 structural analysis and visualize the result [20]. A user 83 can then manually edit the model to improve the stiff${ }_{84}$ ness while getting almost instant feedback. The instant ${ }_{85}$ feedback is only possible because the analysis is limited ${ }_{86}$ to cross-sections. Stava et al. presents a more automated ${ }_{87}$ method for improving stiffness [21]. They perform a ${ }_{88}$ complete worst-case structural analysis on a tetrahedral 89 mesh to determine the structurally weak regions. Based 90 on this analysis, it is decided whether to improve the ${ }_{91}$ model by thickening, hollowing or adding a strut. Fi92 nally, Zhou et al. [22] also perform a worst-case struc${ }_{93}$ tural analysis with more precise determination of the 94 worst-case loads than in [21]. Furthermore, they con${ }_{95}$ clude that solving a shape optimization problem to min${ }_{96}$ imize stress is impractical due to the non-linearity and 
97 non-convexity of the problem. Therefore, they make do 98 with visualizing the structurally weak regions.

99 Topology optimization problems are indeed non100 convex. However, the topology optimization commu101 nity has been solving these problems to at least local 102 optimality for decades and the resulting designs usu${ }_{103}$ ally perform better than designs optimized by humans 104 [2]. Feasible solutions to these problems are often found 105 by standard numerical gradient-based optimization al106 gorithms. However, note that the smooth compliance 107 functional is often chosen as the objective function to 108 ease the optimization instead of the non-smooth, but of109 ten more interesting, maximal stress as Zhou et al. pro110 pose.

111 A key ingredient in a topology optimization method 112 is the shape representation which is required to be able 113 to handle topology changes. Hence, topological opti114 mization has focused primarily on implicit representa115 tions over uniform voxel grids. Such representations ${ }_{116}$ can handle topology changes but lead to fixed-resolution 117 results with cuberille artifacts. The most popular im118 plicit topology optimization approaches are the density 119 and level set approaches. The density approach [23][2] 120 represents the structure by assigning a density value be121 tween 0 (void) and 1 (material) to each cell in a fixed 122 grid or mesh. The structure is now deformed by chang${ }_{123}$ ing these density values. The level set approach uses 124 the level set method [24] evaluated on a fixed grid or 125 mesh [25][26]. Here, the structure is represented by the 126 zero level set and deformed by changes to the level set 127 function. Both methods iteratively change the shape to 128 approach the optimum.

129 We propose to represent the surface explicitly. An ex130 plicit representation, for example a triangle mesh, has ${ }_{131}$ previously been used for shape optimization [9][10]. ${ }_{132}$ However, shape optimization does not allow for topol${ }_{133}$ ogy changes and often only small shape deformations. ${ }_{134}$ Furthermore, it has been used in combination with the 135 level set method [27][28][29][30][31] where it is neces${ }_{136}$ sary to constantly switch between the implicit and ex${ }_{137}$ plicit representations. An explicit representation has 138 also been used in combination with a computationally ${ }_{139}$ expensive remeshing of the entire design domain at each 140 iteration [4][32]. Finally, it has previously been shown ${ }_{141}$ that using the DSC method for topology optimization 142 works in 2D and therefore has potential [33]. However, ${ }_{143}$ here, we show that this concept is able to solve real144 world topology optimization problems in 3D.

145 Note that this list of structural optimization methods 146 is far from exhaustive.

\section{1.2. Contributions}

148 The main contributions of this paper are as follows.

149 - As opposed to previous methods introduced in computer graphics, our method automatically optimizes the shape and topology of a structure given boundary conditions, an objective function, constraints and an initial shape. This completely eliminates the manual editing which has been characteristic for the current approaches.

- Compared to current methods from the topology optimization community, the method uses a single explicit representation to represent the structure and, at the same time, is able to handle topology changes. This gives rise to several advantages including a single mesh for shape representation and finite element calculations, possibility of both continuous and discrete optimization strategies and both the initial and optimized structure are in the form of surface triangle meshes. Finally, the adaptive mesh makes it possible to achieve a much more detailed result within reasonable time on an ordinary laptop than otherwise possible using the standard fixed grid methods.

- To be able to solve real-world topology optimization problems in $3 \mathrm{D}$, it was necessary to make significant changes compared to the 2D proof-ofconcept by Christiansen et al. [33]. Consequently, the discrete step relabels elements based on an optimization procedure which takes constraints into account instead of based on a simple threshold of the objective. Furthermore, the presented method handles self weight, it is initialized by any surface triangle mesh, areas can be fixed to either solid or void and several global constraints have been implemented and utilized. Finally, the requirements for computational efficiency is much higher in 3D than 2D. Therefore, the mesh adaptivity of the DSC method is utilized and the computations are distributed on multiple cores.

\section{${ }_{186}$ 2. Method}

187 The proposed method uses a simplicial complex to 188 represent the shape of a structure. A simplicial com189 plex discretizes a domain into tetrahedral elements. In ${ }_{190}$ 3D it consists of the simplices; nodes (points), edges 191 (line pieces), faces (triangles) and tetrahedra (triangular 192 pyramids). Furthermore, the tetrahedra do not overlap ${ }_{193}$ and any point in the discretized domain is either inside 


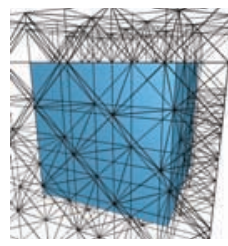

(a) Time step 1

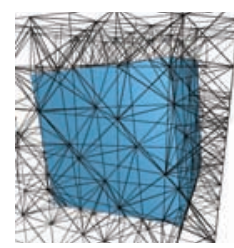

(b) Time step 2

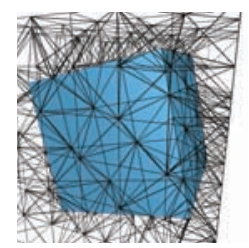

(c) Time step 3
Figure 2: Rotation of a cube using the Deformable Simplicial Complex method. The interface between solid and void (the surface of the cube) is depicted in turquoise. Furthermore, all edges of the simplicial complex are drawn in black.

194 a tetrahedron or on the boundary between tetrahedra. In 195 addition, all tetrahedra are labeled as being either void 196 (no material) or solid (filled with material). Therefore, 197 the interface between solid and void (the surface) is rep198 resented by the faces that are sandwiched between a 199 tetrahedron labeled void and a tetrahedron labeled solid. 200 Figure 2 depicts a cube represented by a simplicial com201 plex. The tetrahedral mesh generator TetGen [34] is 202 used to generate the initial mesh.

203 Apart from the shape representation, the tetrahedral 204 elements of the simplicial complex can be used for 205 physical computations using the finite element method. ${ }_{206}$ Since the finite element analysis will produce large er207 rors if used with nearly degenerate tetrahedra, it is im208 portant to sustain a high quality mesh.

\section{2.1. Deformable Simplicial Complex method}

210 To ensure a high quality mesh, we use the De211 formable Simplicial Complex (DSC) method [3] ${ }^{1}$. The ${ }_{212}$ DSC method ensures high quality tetrahedral elements ${ }_{213}$ during deformation of a model embedded in a simplicial ${ }_{214}$ complex as illustrated in Figure 2. Low quality tetra215 hedra (slivers, wedges, caps and needles) are removed 216 by continuously performing a set of mesh operations 217 while the surface is being deformed. The tetrahedron ${ }_{218}$ quality measure is $\frac{6 \sqrt{2} V}{\left(\frac{1}{6} \sum_{i} l_{i}^{2}\right)^{3 / 2}}$ [35] where $V$ is the volume ${ }_{219}$ of the tetrahedron and $l_{i}$ is the length of edge $i$. Note 220 that the DSC method only improves the mesh quality 221 where necessary (often near the surface). Furthermore, 222 the DSC method also handles topology changes by re223 moving low quality tetrahedra which are sandwiched 224 between two surfaces. This is illustrated by two objects 225 colliding in Figure 3.

\footnotetext{
${ }^{1} \mathrm{An}$ open-source framework is available at www.github.com/ asny/DSC
}

(a) Time step 1

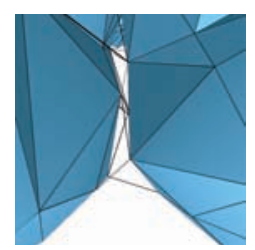

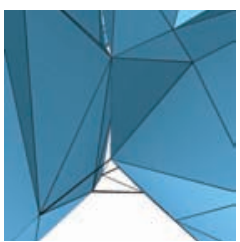

(b) Time step 2

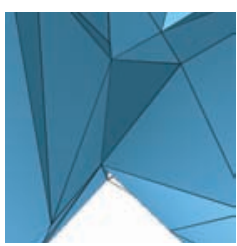

(c) Time step 3
Figure 3: Illustration of topology changes using the Deformable Simplicial Complex method. Here, only edges having both end nodes on the surface are drawn. As the objects approach each other the tetrahedra between the objects get squeezed. When a tetrahedron between the two surfaces is squeezed too much, this tetrahedron will be collapsed. Consequently, the only thing separating the two objects is a face. However, this face has tetrahedra which are labeled solid on both sides and it is therefore no longer part of the surface. Consequently, the two objects are now merged into one.

226 In addition to ensuring high quality tetrahedral ele227 ments, the DSC method also controls the level of detail 228 of both the surface and the tetrahedral mesh. In prac229 tice, the DSC method attempts to collapse too small 230 simplices and split too large simplices. Consequently, ${ }_{231}$ we always attain a mesh of the desired complexity, de${ }_{232}$ scribed by the discretization parameter $\delta$ (corresponding 233 to the average edge length). More importantly, the de234 tail control allows for mesh adaptivity. This means that 235 smooth regions on the surface are represented by a more 236 coarse discretization than regions with small features.

${ }_{237}$ The mesh operations used are smoothing [36] (not ${ }_{238}$ performed on surface nodes), edge split [37], edge col${ }_{239}$ lapse [37], edge removal [38] and multi-face removal 240 [38]. The latter two use the flips illustrated in Figure 2414 . Consequently, these two mesh operations do not 242 change the position of any nodes, only the connectiv${ }_{243}$ ity. The quality of the mesh is improved by all five 244 operations, whereas the detail level of the mesh is con245 trolled through the operations edge split and edge col246 lapse. Note that changes have been made compared to 247 [3]. The multi-face retriangulation, optimization-based 248 smoothing, null-space smoothing and tetrahedron rela249 beling operations have not been necessary for this ap250 plication. Removing these operations has resulted in a 251 significant speed-up. Also, the edge removal operation 252 on the surface and boundary is an addition since [3].

${ }_{253}$ The strategy for moving the surface nodes is to first ${ }_{254}$ compute a destination $p_{n}^{*}$ for each surface node $n$ cur255 rently at position $p_{n}$. The destination $p_{n}^{*}$ is computed us256 ing a user-defined velocity function which, for the case 257 of topology optimization, will be described later. After258 wards, all surface nodes are moved from $p_{n}$ to $p_{n}^{*}$ using 259 the strategy illustrated in Figure 5. 


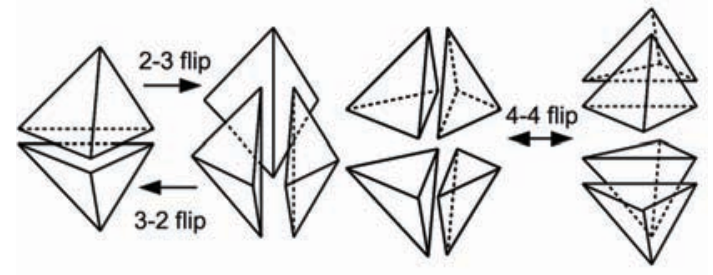

Figure 4: Illustrations of 2-3, 3-2 and 4-4 flips inspired by the illustration in [38].

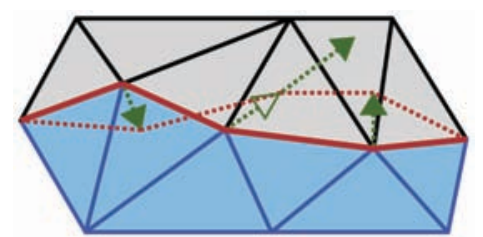

Figure 5: Illustration of how the surface (red) is moved in 2D. The same principle applies to 3D. A filled arrow indicates the destination $\boldsymbol{p}_{n}^{*}$ of the surface node $n$. One of the nodes cannot move to its destination without creating low quality tetrahedra and it is therefore only moved as depicted by the unfilled arrow. The other two are moved to their destinations. Then, mesh operations are applied to improve the mesh quality and the node that did not reach its destination is moved again. This is repeated until all nodes have reached their destinations.

\section{2.2. Structural analysis}

261 In this paper, we will optimize the topology of phys262 ically valid structures in static equilibrium. In order 263 to achieve physical validity, structural analyses using 264 the finite element method are performed. This implies 265 considering the discretization, boundary conditions and ${ }_{266}$ equilibrium which are the topics of this section.

${ }_{267}$ As described previously, a domain is discretized into 268 high quality tetrahedral elements which are analyzed 269 using the finite element method. Using quadratic ba270 sis functions solves a well-known issue with a jagged 271 surface when using the analysis as a basis for non272 parametric shape optimization [11][12]. Consequently, 273 quadratic basis functions are chosen instead of linear 274 to interpolate the tetrahedral elements. Therefore one 275 control point $c$ is associated with each node and edge 276 of a tetrahedron. Furthermore, the positions of all ${ }_{277}$ control points are assembled in a vector termed $\boldsymbol{p}=$ $278\left[\ldots, \boldsymbol{p}_{c}^{T}, \ldots\right]^{T}$. In addition, each tetrahedron $t$ has an 279 associated material $m_{t}$ with material parameters density ${ }_{280} \rho_{t}$, Young's modulus $E_{t}$ and Poisson's ratio $v_{t}$. Finally, ${ }_{281}$ the materials of the tetrahedra are also assembled in a 282 vector $\boldsymbol{m}=\left[\ldots, m_{t}, \ldots\right]^{T}$.

${ }_{283}$ The local stiffness matrix $\boldsymbol{K}_{t}$ contains information on 284 the stiffness of tetrahedron $t$. It depends on both the 285 positions of the control points $\boldsymbol{p}$ and the materials of the
286 tetrahedra $\boldsymbol{m}$ and can be calculated by

$$
\boldsymbol{K}_{t}(\boldsymbol{m}, \boldsymbol{p})=\int_{V_{t}} \boldsymbol{B}_{t}^{T}(\boldsymbol{p}) \boldsymbol{E}_{t}(\boldsymbol{m}) \boldsymbol{B}_{t}(\boldsymbol{p}) \partial(x, y, z)
$$

${ }_{287}$ We have chosen only to consider isotropic linear materi288 als. Consequently, the constitutive matrix $\boldsymbol{E}_{t}(\boldsymbol{m})$ which 289 relates stress and strain is

$$
\boldsymbol{E}=\frac{E}{(1+v)(1-2 v)}\left[\begin{array}{cccccc}
1-v & v & v & 0 & 0 & 0 \\
v & 1-v & v & 0 & 0 & 0 \\
v & v & 1-v & 0 & 0 & 0 \\
0 & 0 & 0 & \frac{1-2 v}{2} & 0 & 0 \\
0 & 0 & 0 & 0 & \frac{1-2 v}{2} & 0 \\
0 & 0 & 0 & 0 & 0 & \frac{1-2 v}{2}
\end{array}\right]
$$

290 where $\boldsymbol{E}_{t}(\boldsymbol{m})$ is shortened to $\boldsymbol{E}, E_{t}(\boldsymbol{m})$ to $E$ and $v_{t}(\boldsymbol{m})$ 291 to $v$. Finally, the strain-displacement matrix $\boldsymbol{B}_{t}(\boldsymbol{p})$ is re292 lated to the shape of the tetrahedron and the basis func293 tions. For more details, see a text book on the finite ele294 ment method used for structural analysis, e.g. [39]. The 295 global stiffness matrix $\boldsymbol{K}(\boldsymbol{m}, \boldsymbol{p})$ can then be assembled 296 from the local stiffness matrices $\boldsymbol{K}_{t}(\boldsymbol{m}, \boldsymbol{p})$. Note that for 297 elements with void as the associated material, $\boldsymbol{K}_{t}$ is not 298 defined. Consequently, the void elements are eliminated 299 from the finite element analysis, which decreases com300 putation time.

301 In this paper, we will limit ourselves to static prob${ }_{302}$ lems subject to a single load case. These problems are 303 modeled by supports and external forces $\boldsymbol{f}_{c}$ which are 304 both applied to the surface of the structure. In addition 305 to external forces, the weight of the structure will cause 306 gravitational forces

$$
\boldsymbol{w}_{c}(\boldsymbol{m}, \boldsymbol{p})=\boldsymbol{g} \sum_{i \in c} a_{i} \rho_{i}(\boldsymbol{m}) \quad V_{i}(\boldsymbol{p})
$$

${ }_{307}$ Here, $\boldsymbol{g}=[0,-9.8,0]^{T} \mathrm{~m} / \mathrm{s}^{2}$ is a vector of the gravita${ }_{308}$ tional acceleration and $a_{i}$ is a scale factor computed by a 309 mass lumping scheme for each element $i$. Furthermore, ${ }_{310} \rho_{i}$ is the density and $V_{i}(\boldsymbol{p})$ is the volume of tetrahedral ${ }_{311}$ element $i$ which is adjacent to control point $c$. Conse312 quently, the global force vector is

$$
\boldsymbol{f}(\boldsymbol{m}, \boldsymbol{p})=\left[\ldots, \boldsymbol{f}_{c}^{T}+\boldsymbol{w}_{c}^{T}(\boldsymbol{m}, \boldsymbol{p}), \ldots\right]^{T}
$$

${ }_{313}$ Since we desire a structure in static equilibrium, the 314 Sum of the forces on all particles must be zero (New315 ton's first law). Consequently, we will utilize the equi316 librium equations

$$
K(m, p) u=f(m, p)
$$

${ }_{317}$ These equations are used to calculate the global dis${ }_{318}$ placement vector $\boldsymbol{u}=\left[\ldots, \boldsymbol{u}_{c}, \ldots\right]$. At each control 
319 point $c, \boldsymbol{u}_{c}$ represents the displacement caused by the 320 forces $\boldsymbol{f}$ applied to the structure. Note that, since $\boldsymbol{K}$ and ${ }_{321} \boldsymbol{f}$ are functions of $\boldsymbol{p}$ and $\boldsymbol{m}$, so is $\boldsymbol{u}$.

${ }_{322}$ Solving the equilibrium equations is the most time ${ }_{323}$ consuming part of the optimization. Furthermore, the 324 number of equations scales linearly with the number 325 of degrees of freedom. Consequently, the sparse solver ${ }_{326}$ CHOLMOD [40], which is a part of the SuiteSparse li327 brary [41], is used to solve the equilibrium equation ef328 ficiently using multiple cores.

\section{2.3. Optimization}

${ }_{330}$ We want to optimize an objective function $f$ by ${ }_{331}$ changing the shape and topology of the structure. ${ }_{332}$ Therefore, the objective can be anything as long as it ${ }_{33}$ is a function of the shape and topology. Furthermore, ${ }_{334}$ there are two ways to change the shape and topology. ${ }_{335}$ The first is to change the position $\boldsymbol{p}_{n}$ of a design node ${ }_{336} n$, the other is to change the material $m_{e}$ of a design ele${ }_{337}$ ment $e$. A node is a design node $n$ if it is

- on the surface of the structure,

- not supported,

- not subjected to any external forces and

- not part of a fixed domain (see Section 2.5).

${ }_{342}$ Furthermore, a tetrahedral element is a design element ${ }_{343} e$ if it is

344 - solid,

345

- not adjacent to a control point subjected to external forces and

- not part of a fixed domain (see Section 2.5).

For the test cases presented here, we seek to find the 349 structure which is as stiff as possible. Consequently, the ${ }_{350}$ objective function is compliance

$$
f(\boldsymbol{m}, \boldsymbol{p})=\boldsymbol{u}^{T} \boldsymbol{K}(\boldsymbol{m}, \boldsymbol{p}) \boldsymbol{u}
$$

${ }_{351}$ Note that since this objective is a function of the dis${ }_{352}$ placements $\boldsymbol{u}$, we need to solve Equation 4 to evaluate ${ }_{353}$ it. The reason for choosing to minimize compliance and 354 not for example maximal Von Mises stress is that the 355 compliance function is smooth. This is a significant ad356 vantage for the optimization algorithm. However, we ${ }_{357}$ plan to minimize the maximal Von Mises stress using 358 the same method in the future.

359 It is often desirable to constrain the optimization. In 360 some test examples, we choose to limit the amount of
361 material used, i.e. the optimization is subject to a global 362 volume constraint:

$$
g_{1}(\boldsymbol{m}, \boldsymbol{p})=\frac{V(\boldsymbol{m}, \boldsymbol{p})}{V^{*}}-1
$$

${ }_{363}$ Where $V(\boldsymbol{m}, \boldsymbol{p})$ is the total volume of the solid elements 364 and $V^{*}$ is the maximum volume of the structure.

365 Optimized results are often not manufacturable. For ${ }_{366}$ example, the optimized results often contain many de367 tails. A partial remedy is to constrain the total surface 368 area, called a perimeter constraint [42].

$$
g_{2}(\boldsymbol{m}, \boldsymbol{p})=\frac{A(\boldsymbol{m}, \boldsymbol{p})}{A^{*}}-1
$$

${ }_{369}$ Here, $A(\boldsymbol{p})$ is the total area of triangles sandwiched be370 tween a void and a (not fixed) solid element and $A^{*}$ is ${ }_{371}$ the maximum surface area allowed. This constraint en372 forces a smoothness of the surface and thereby to some 373 degree prevents small details and thin plates. However, 374 since it is a global constraint, these undesirable features 375 are not guaranteed to be eliminated.

376 Finally, in some cases, we want to limit the possible 377 change from the original shape. In these cases, the orig378 inal design nodes are added to a set $O$. If, during the 379 optimization, an edge connecting two original nodes is 380 split, the new node will be added to the set. However, 381 if a hole appears inside the structure, the nodes on that 382 internal surface are not added. Furthermore, the origi383 nal surface is stored such that the distance $d_{n}(\boldsymbol{m}, \boldsymbol{p})$ from ${ }_{384} n \in O$ to the original surface can be calculated. Finally, 385 the function $t_{n}(\boldsymbol{m}, \boldsymbol{p})$ computes the distance from $n \notin O$ ${ }_{386}$ to the surface represented by the nodes in the set $O$. In 387 other words, this function calculates the thickness of the ${ }_{388}$ shell of the structure. We can now limit the change from 389 the original surface as well as ensuring that holes will 390 not appear in this surface by applying the constraint:

$$
\begin{aligned}
g_{3}(\boldsymbol{m}, \boldsymbol{p}) & =\frac{1}{N_{\in O}} \sum_{n \in O} \max \left(d_{n}(\boldsymbol{m}, \boldsymbol{p})-D^{*}, 0\right)^{2} \\
& +\frac{1}{N_{\notin O}} \sum_{n \notin O} \max \left(T^{*}-t_{n}(\boldsymbol{m}, \boldsymbol{p}), 0\right)^{2}
\end{aligned}
$$

${ }_{391}$ Here, $D^{*}$ is the maximal change from the original sur392 face and $T^{*}$ is the minimum thickness of the shell of 393 the structure. Note that $g_{3}$ is $C^{1}$ continuous and thereby 394 differentiable.

\section{2.3.1. Continuous optimization}

396 The first part of the optimization procedure is to lo397 cally perturb the surface of the structure such that it it398 eratively gets closer to optimum. This part of the opti399 mization procedure consists of calculating an improved 


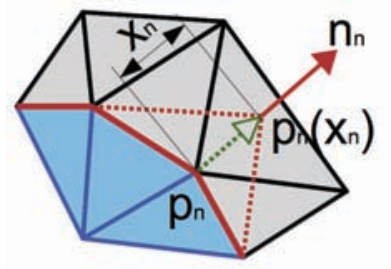

Figure 6: Illustrates the destination $\boldsymbol{p}_{n}\left(x_{n}\right)$ of node $n$ as a function of the design variable $x_{n}$. Furthermore, $\boldsymbol{p}_{n}$ is the current position and $\boldsymbol{n}_{n}$ is the normal.

400 position $\boldsymbol{p}_{n}^{*}$ for each design node $n$. Afterwards, the 401 structure is deformed by moving each design node from ${ }_{402}$ its current $\boldsymbol{p}_{n}$ to the more optimal position $\boldsymbol{p}_{n}^{*}$ as de403 scribed in Section 2.1. Note that since the DSC method 404 handles topology changes, these can occur. Thin struc405 tures can collapse and holes can disappear. However, 406 holes will not appear inside the structure during this ${ }_{407}$ step. Also, note that the material parameter $\boldsymbol{m}$ is fixed 408 during this step.

409 Moving the design nodes in the tangent directions 410 will not change the surface much. Consequently, each ${ }_{411}$ design node $n$ is associated with one design variable ${ }_{412}$ only. A design variable $x_{n}$ represents the distance node $n$ ${ }_{413}$ is moved in the normal direction $\boldsymbol{n}_{n}$ from the current po${ }_{414}$ sition $\boldsymbol{p}_{n}$ as illustrated in Figure 6. The design variables 415 are assembled in the vector $\boldsymbol{x}=\left[\ldots, x_{n}, \ldots\right]^{T}$. Conse${ }_{416}$ quently, the positions of the control points as a function ${ }_{417}$ of the design variables can be expressed as $\boldsymbol{p}(\boldsymbol{x})$.

${ }_{418}$ The relation between the current position $\boldsymbol{p}_{n}$, the op${ }_{419}$ timized position $\boldsymbol{p}_{n}^{*}$ and the optimized design variable $x_{n}^{*}$ 420 for a design node $n$ is

$$
\boldsymbol{p}_{n}^{*}=\boldsymbol{p}_{n}\left(x_{n}^{*}\right)=\boldsymbol{p}_{n}+x_{n}^{*} \boldsymbol{n}_{n}
$$

${ }_{421}$ To estimate $\boldsymbol{x}^{*}=\left[\ldots, x_{n}^{*}, \ldots\right]^{T}$, a smooth non-linear op422 timization problem is solved:

$$
\begin{aligned}
\boldsymbol{x}^{*}=\underset{\boldsymbol{x}}{\arg \min } & : f(\boldsymbol{m}, \boldsymbol{p}(\boldsymbol{x}))=\boldsymbol{u}^{T} \boldsymbol{K}(\boldsymbol{m}, \boldsymbol{p}(\boldsymbol{x})) \boldsymbol{u} \\
\text { subject to } & : g_{i}(\boldsymbol{m}, \boldsymbol{p}(\boldsymbol{x})) \leq 0, i=1,2,3 \\
& : \boldsymbol{K}(\boldsymbol{m}, \boldsymbol{p}(\boldsymbol{x})) \boldsymbol{u}=\boldsymbol{f}(\boldsymbol{m}, \boldsymbol{p}(\boldsymbol{x})) \\
& : \boldsymbol{x}^{\min } \leq \boldsymbol{x} \leq \boldsymbol{x}^{\max }
\end{aligned}
$$

${ }_{423}$ Here, $\boldsymbol{x}^{\min }=\left[\ldots, x_{n}^{\min }, \ldots\right]^{T}$ and $\boldsymbol{x}^{\max }=\left[\ldots, x_{n}^{\max }, \ldots\right]^{T}$ ${ }_{424}$ are move limits on the design variables $\boldsymbol{x}$. Generally, ${ }_{425} \boldsymbol{x}^{\min }$ and $\boldsymbol{x}^{\max }$ are chosen such that the design nodes 426 will not create degenerate tetrahedra during the opti${ }_{427}$ mization. Consequently, the new shape can only be a ${ }_{428}$ small perturbation from the current shape and Equation ${ }_{429} 10$ will be solved many times. Furthermore, the move
${ }_{430}$ limits ensure that the design nodes stay inside a user${ }_{431}$ specified design domain. Therefore, the structure can432 not extend beyond the boundaries of this design domain. ${ }_{433}$ We use the gradient-based optimization algorithm ${ }_{434}$ Method of Moving Asymptotes (MMA) [14] to solve 435 the optimization problem in Equation 10. This is an iter${ }_{436}$ ative optimization procedure which is stopped when the ${ }_{437}$ infinity norm of the change in $\boldsymbol{x}$ is less than a threshold 438 or at iteration 5. In addition to evaluating the objective ${ }_{439}$ function and constraints, the derivatives of these func${ }_{440}$ tions with respect to each of the design variables $x_{n}$ have ${ }_{441}$ to be evaluated at each iteration. Computing $\frac{\partial}{\partial x_{n}} \boldsymbol{u}$ is not 442 efficient. However, using the adjoint variable method ${ }_{443}$ (utilizing the equilibrium equations) [43][44], we get 444 an analytical expression for $\frac{\partial}{\partial x_{n}} f(\boldsymbol{m}, \boldsymbol{p}(\boldsymbol{x}))$ without the ${ }_{445}$ problematic term $\frac{\partial}{\partial x_{n}} \boldsymbol{u}$ :

$$
\begin{aligned}
& \frac{\partial f(\boldsymbol{m}, \boldsymbol{p}(\boldsymbol{x}))}{\partial x_{n}}= \\
& -\boldsymbol{u}^{T} \frac{\partial \boldsymbol{K}(\boldsymbol{m}, \boldsymbol{p}(\boldsymbol{x}))}{\partial x_{n}} \boldsymbol{u}+2 \boldsymbol{u}^{T} \frac{\partial \boldsymbol{f}(\boldsymbol{m}, \boldsymbol{p}(\boldsymbol{x}))}{\partial x_{n}}
\end{aligned}
$$

${ }_{446}$ Still, since the equilibrium equations have to be evalu${ }_{447}$ ated at each iteration, this continuous optimization step ${ }_{448}$ is the most expensive part of the optimization proce449 dure.

\section{2.3.2. Discrete optimization}

451 In addition to changing the shape by moving the de452 sign nodes, a discrete optimization step is performed ${ }_{453}$ where the materials $\boldsymbol{m}$ are changed and the positions $\boldsymbol{p}$ ${ }_{454}$ are not. The step has two purposes; introducing holes 455 inside the structure and increasing the convergence rate 456 of the continuous optimization. The optimization prob457 lem can be written as

$$
\begin{aligned}
\boldsymbol{m}^{*}=\underset{\boldsymbol{m}}{\arg \min } & : f(\boldsymbol{m}, \boldsymbol{p})=\boldsymbol{u}^{T} \boldsymbol{K}(\boldsymbol{m}, \boldsymbol{p}) \boldsymbol{u} \\
\text { subject to } & : g_{i}(\boldsymbol{m}, \boldsymbol{p}) \leq 0, i=1,2,3 \\
& : \boldsymbol{K}(\boldsymbol{m}, \boldsymbol{p}) \boldsymbol{u}=\boldsymbol{f}(\boldsymbol{m}, \boldsymbol{p}) \\
& : m_{e} \in\{\text { void, solid }\}
\end{aligned}
$$

${ }_{458}$ Note that the set of possible materials is limited to void 459 and solid. However, it is possible to extend this ap${ }_{460}$ proach to handle multiple materials. Furthermore, we 461 choose that only solid elements are design elements. ${ }_{462}$ Consequently, this step only removes material from the ${ }_{463}$ structure. If it removes material near the surface, this ${ }_{464}$ will speed up shape changes. On the other hand, if it ${ }_{465}$ removes material inside the structure, a hole is created. 
${ }_{466}$ The discrete optimization problem in Equation 12 is ${ }_{467}$ NP-hard. However, since this optimization problem is ${ }_{468}$ combined with a continuous optimization, it is not nec${ }_{469}$ essary to solve it to optimality. Consequently, this step 470 will seek to improve the objective while trying to satisfy 471 the constraints by relabeling tetrahedra. The relabeling 472 will be based on discrete derivatives, i.e. the change in 473 objective or constraints when changing the material in ${ }_{474}$ element $e$ from solid to void:

$$
\begin{gathered}
\Delta_{e} f(\boldsymbol{m}, \boldsymbol{p})=f\left(\boldsymbol{m}_{e}^{v}, \boldsymbol{p}\right)-f(\boldsymbol{m}, \boldsymbol{p}) \\
\Delta_{e} g_{i}(\boldsymbol{m}, \boldsymbol{p})=g_{i}\left(\boldsymbol{m}_{e}^{v}, \boldsymbol{p}\right)-g_{i}(\boldsymbol{m}, \boldsymbol{p}), i=1,2,3
\end{gathered}
$$

${ }_{475}$ Here, $\boldsymbol{m}_{e}^{v}$ equals $\boldsymbol{m}$ where $m_{e}$ is void instead of solid. 476 However, computing these discrete derivatives for com477 pliance is inefficient since the equilibrium equations 478 then have to be evaluated once for each solid tetrahe479 dron. Instead, we will use an approximation based on 480 the theory of topological derivatives [4][5][45][6]. The ${ }_{481}$ topological derivative corresponds to the influence on ${ }_{482}$ the objective function of introducing an infinitesimal ${ }_{483}$ hole in element $e$. For compliance, the discrete deriva484 tive can therefore be approximated by

$$
\Delta_{e} f(\boldsymbol{m}, \boldsymbol{p}) \approx 3 \boldsymbol{u}^{T} \boldsymbol{K}_{e}(\boldsymbol{m}, \boldsymbol{p}) \boldsymbol{u}-\frac{2 V_{e}(\boldsymbol{p})}{N_{\epsilon e}} \sum_{c \in e} \boldsymbol{u}_{c}^{T} \boldsymbol{g}
$$

485 The first part of the optimization strategy is to im${ }_{486}$ prove the objective function while decreasing or satis${ }_{487}$ fying all constraints. A constraint $i$ is decreased if

$$
\Delta_{e} g_{i}(\boldsymbol{m}, \boldsymbol{p}) \leq 0
$$

488 and satisfied if

$$
g_{i}(\boldsymbol{m}, \boldsymbol{p})+\Delta_{e} g_{i}(\boldsymbol{m}, \boldsymbol{p}) \leq 0
$$

489 Hence, a design element $e$ is relabeled from solid to void 490 if either of equations 16 and 17 are satisfied for all con491 straints and

$$
\Delta_{e} f(\boldsymbol{m}, \boldsymbol{p})<0
$$

492 The second part of the optimization is to try to im493 prove constraints which are not satisfied. Therefore, if 494 constraint $i$ is not satisfied, i.e. $g_{i}(\boldsymbol{m}, \boldsymbol{p})>0$, we will 495 try to find an optimal design element $e^{*}$ to relabel from 496 solid to void. Noting that $\Delta_{e} f(\boldsymbol{m}, \boldsymbol{p}) \geq 0$, the optimal 497 design element $e^{*}$ is found by solving

$$
e^{*}=\underset{e}{\arg \min }-\frac{\Delta_{e} f(\boldsymbol{m}, \boldsymbol{p})}{\Delta_{e} g_{i}(\boldsymbol{m}, \boldsymbol{p})}
$$

498 where all arguments $e$ satisfy

$$
\Delta_{e} g_{i}(\boldsymbol{m}, \boldsymbol{p})<0
$$

499 and either Equation 16 or 17 for all constraints. Design 500 element $e^{*}$ is then relabeled from solid to void. This 501 process is repeated as long as constraint $i$ is not satisfied 502 and an optimal element $e^{*}$ exists.

\section{2.4. Disconnected material}

504 The continuous and discrete optimization steps can 505 very well result in material which is disconnected from 506 the main structure. These parts do not contribute to the 507 objective. Furthermore, since void elements are elimi508 nated from the finite element analysis, disconnected ma509 terial will result in the equilibrium equations not having 510 a unique solution. Consequently, disconnected mate${ }_{511}$ rial is removed by performing a connected component 512 analysis and making every component, except for the 513 largest, void.

\section{2.5. Initialization}

515 To initialize the optimization, the user has to specify 516 boundary conditions, an objective function, constraints 517 and an initial structure.

${ }_{518}$ The boundary conditions are the supports and exter519 nal forces applied to the surface of the structure as de520 scribed in Section 2.2. Furthermore, the boundaries of 521 the design domain (the domain where material can re522 side) have to be specified. Finally, it is possible to spec523 ify fixed domains (areas that are either always solid or 524 always void). The fixed void areas are implemented as 525 not being a part of the design domain. However, the 526 fixed solid domains are enforced by assigning a differ${ }_{527}$ ent label to the tetrahedra inside these domains. Con528 sequently, an invisible surface exists between the fixed 529 and non-fixed solid domains. The shape of this surface ${ }_{530}$ should not be changed in any way. However, we still ${ }_{531}$ want the DSC method to improve the mesh quality and ${ }_{532}$ control the level of detail at this surface. Consequently, 533 the DSC method is modified such that only mesh oper${ }_{534}$ ations which do not change the surface are performed at ${ }_{535}$ the surface between fixed and non-fixed domains.

${ }_{536}$ In all of the example problems presented here, the ob${ }_{537}$ jective is to minimize compliance since it is often desir${ }_{538}$ able to produce as stiff a structure as possible. However, 539 choosing another objective is as simple as changing the 540 objective function and calculating the shape and topo${ }_{541}$ logical derivatives of the new function. For example, ${ }_{542}$ the same approach has been used for balancing of 3D 543 models [46]. Furthermore, different problems require ${ }_{544}$ different constraints. In this paper, we present several 545 different global constraints to illustrate their effect on 
${ }_{546}$ the design. The effect can be quite drastic and conse547 quently the constraints are as important as the objective. ${ }_{548}$ Finally, the initial model is a triangle mesh. Conse${ }_{549}$ quently, any surface mesh can be used as a starting point 550 for the optimization without any conversions. In this pa${ }_{551}$ per, we choose to initialize the optimization by triangle 552 meshes of existing models and by generated meshes that ${ }_{553}$ fill the entire design domain.

\section{2.6. Method summary}

555 The method consists of two steps:

\section{${ }_{556}$ Step 1: Discrete optimization}

\section{${ }_{562}$ Step 2: Continuous optimization}

592 the optimization is initialized by a 3D model, the opti593 mum is assumed to be close. However, that is proba594 bly not the case when the optimization is initialized by 595 filling the design domain with material. Consequently, ${ }_{596}$ in these cases, we slowly lower the discretization pa${ }_{597}$ rameter $\delta$ by multiplying it by 0.99 at each time step. 598 The detail control, described in Section 2.1, will then 599 increase the mesh complexity. Note that this strategy 600 is especially effective since the method only calculates 601 on solid elements. However, solving the equilibrium 602 equations is still the most time-consuming part. Conse603 quently, we utilize multiple threads on the CPU to speed 604 up these computations. Also, computing the gradients 605 of the compliance function and assembling the global 606 stiffness matrix $K$ and force vector $F$ are parallelized.

\section{3. Results}

608 The proposed method can be used in the fabrication 609 design process in areas such as construction, manufac610 turing and design. In this section, we will illustrate ${ }_{611}$ this statement by solving problems within each of these ${ }_{612}$ fields. The results are generated on a laptop with a 2.4 ${ }_{613} \mathrm{GHz}$ quad-core Intel Core $i 7$ processor and $8 \mathrm{~GB}$ of 6141333 MHz DDR3 RAM. Parameters and performance 615 measures are depicted in Table 1. Furthermore, the ob616 jective of all examples is to minimize compliance sub617 ject to constraints as depicted in Table 1 .

${ }_{618}$ The raw surface triangle meshes of the optimized 619 structures, i.e. the output as it looks from the optimiza620 tion method, are visualised using Blender. No post pro${ }_{621}$ cessing like subdivision and smoothing has been uti622 lized to improve the appearance. Furthermore, when ${ }_{623}$ material has been removed from inside a structure, the 624 internal cavities are visualized by making the structure 625 transparent. In addition to the optimized result, we will 626 in some cases visualize the strain energy density (SED) ${ }_{627}$ at the surface of the final model. The SED depicts how ${ }_{628}$ much strain an element at the surface is subjected to. ${ }_{629}$ Here, the jet colormap is used, where blue and red de${ }_{630}$ pict low and high SED respectively. Furthermore, the ${ }_{631}$ SEDs are scaled between the minimum and maximum ${ }_{632}$ SED of the initial structure. Consequently, this visual${ }_{633}$ izes how the stiffness has changed as a consequence of ${ }_{634}$ the optimization. In the same cases, we will also visual${ }_{635}$ ize the difference from the original model by a grayscale ${ }_{636}$ colormap. Here, gray means no change, darker means it ${ }_{637}$ has moved in the negative normal direction and lighter ${ }_{638}$ that it has moved in the normal direction. The distance ${ }_{639}$ is scaled by the largest change. 


\begin{tabular}{|c||c|c|c|c|c|c|c|c|c|}
\hline Problem & $\delta$ & $V^{*}(\alpha)$ & $A^{*}(\beta)$ & $D^{*}$ & $T^{*}$ & $f^{*} / f^{0}$ & Surface & Complex & Running time \\
\hline & $\mathrm{mm}$ & $\% V^{0}(-)$ & $\% A^{0}(-)$ & $\% \delta$ & $\% \delta$ & - & \# faces & \# elements & minutes (\#) \\
\hline \hline Bridge & 423 & $20(0.96)$ & $30(0.98)$ & - & - & $304 \%$ & 9883 & 29836 & $68(70)$ \\
\hline Statue & 50 & $50(0.95)$ & - & 15 & 100 & $27 \%$ & 35868 & 66314 & $275(20)$ \\
\hline Dinosaur & 1.4 & - & - & 15 & 100 & $46 \%$ & 6876 & 15071 & $11(5)$ \\
\hline Armadillo & 2.8 & - & - & 15 & 100 & $13 \%$ & 9872 & 15819 & $60(50)$ \\
\hline Table 1 & 42 & $15(0.96)$ & $30(0.98)$ & - & - & $2671 \%$ & 5492 & 11761 & $16(100)$ \\
\hline Table 2 & 62 & $15(0.96)$ & $35(0.98)$ & - & - & $964 \%$ & 3543 & 5521 & $13(60)$ \\
\hline Table 3 & 42 & $15(0.96)$ & $30(0.98)$ & - & - & $5929 \%$ & 5374 & 11759 & $20(100)$ \\
\hline Chair 1 & 21 & $12.5(0.96)$ & $25(0.98)$ & - & - & $1199 \%$ & 4413 & 7929 & $15(100)$ \\
\hline Chair 2 & 21 & $12.5(0.96)$ & $30(0.98)$ & - & - & $625 \%$ & 5527 & 9026 & $18(100)$ \\
\hline Chair 3 & 27 & $12.5(0.96)$ & $30(0.98)$ & - & - & $927 \%$ & 3382 & 4927 & $8(75)$ \\
\hline Support & 655 & $20(0.96)$ & $20(0.98)$ & - & - & $17 \%$ & 15064 & 27120 & $109(100)$ \\
\hline
\end{tabular}

Table 1: Method parameters and performance measures for all example problems. The displayed values are the values as they appear after the optimization. The $V^{*}$ and $A^{*}$ values are stated in percent of the initial volume $V^{0}$ and surface area $A^{0}$ respectively whereas $D^{*}$ and $T^{*}$ are stated in percent of the discretization parameter $\delta$. Furthermore, $f^{0}$ and $f^{*}$ are initial and final compliance respectively. Finally, the \# in the right-most column is the number of time steps.

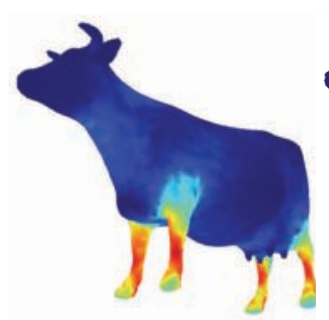

(a) Initial SED

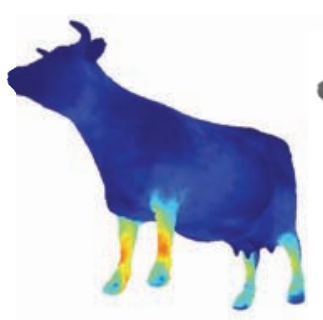

(b) Final SED

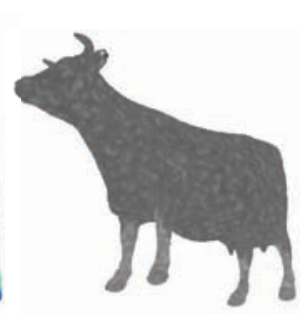

(c) Change

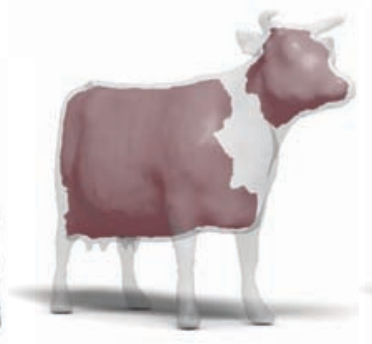

(d) Transparent statue

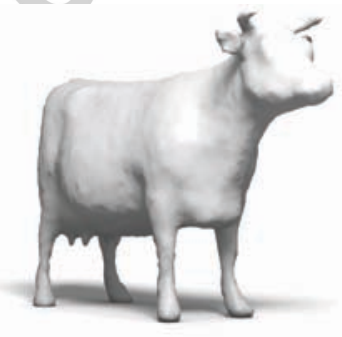

(e) Statue

Figure 7: Topology optimized cow statue which show that the method can optimize stiffness while saving material.

\section{3.1. Construction}

641 Topology optimization has traditionally been used 642 for construction where the objective is to save material 643 while ensuring stiffness. The presented method has the ${ }_{644}$ same capabilities as previous methods. Furthermore, it ${ }_{645}$ extends those methods by being able to initialize an op${ }_{646}$ timization by a surface triangle mesh with no conversion 647 necessary.

${ }_{648}$ First, a bridge problem is initialized by a steel cube ${ }_{649}\left(\begin{array}{lll}30 & 15 & 12 \mathrm{~m}^{3}\end{array}\right)$ with a space for vehicles and supports 650 as depicted in Figure 1. The surface of the bridge is 651 fixed and subjected to a distributed load pushing down652 wards $(100 \mathrm{MPa})$. The result and optimization process ${ }_{653}$ are also depicted in Figure 1. The result shows that com${ }_{654}$ pliance has increased to $304 \%$ of the initial value during 655 the optimization process. However, the optimized struc${ }_{656}$ ture only uses $20 \%$ of the material used by the initial 657 structure.

658 Next, a 4 m-long concrete statue is initialized by a 659 3D model of a cow (source: Aim@Shape). The statue
660 is solid concrete, only subjected to gravitational forces 661 and supported underneath all of its hoofs. The change 662 in SED, shape changes and the optimized cow statue ${ }_{663}$ are depicted in Figure 7. This example shows that our ${ }_{664}$ method extends previous methods by being able to ini${ }_{665}$ tialize an optimization by a 3D model (represented by ${ }_{666}$ a triangle mesh) without any conversion and, further667 more, remain close to this shape. Also, since the statue 668 is subjected to gravitational forces only, compliance is 669 improved at the same time as the amount of material is 670 reduced.

\section{3.2. Manufacturing}

672 An important application of our method is as a tool to 673 improve the stiffness of a given shape. Assume, we are ${ }_{674}$ given a $3 \mathrm{D}$ shape that is to be fabricated. The problem 675 is to change the exterior shape as little as possible while 676 using a minimum amount of material and ensuring that 677 the fabricated object will be able to support itself and 678 moreover withstand specified external loads. Further- 


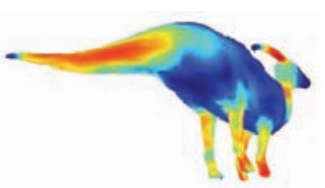

(a) Initial SED

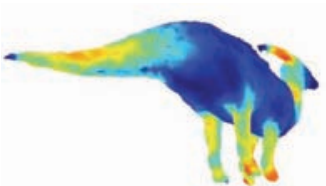

(b) Final SED

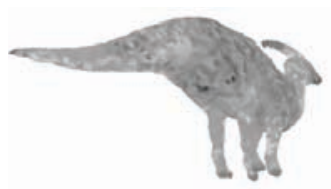

(c) Change

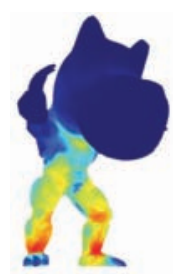

(d) Initial SED

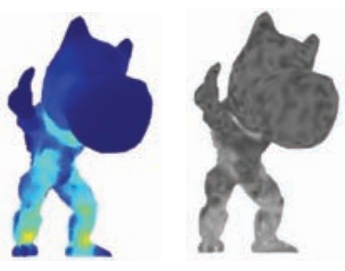

(e) Final SED

(f) Change

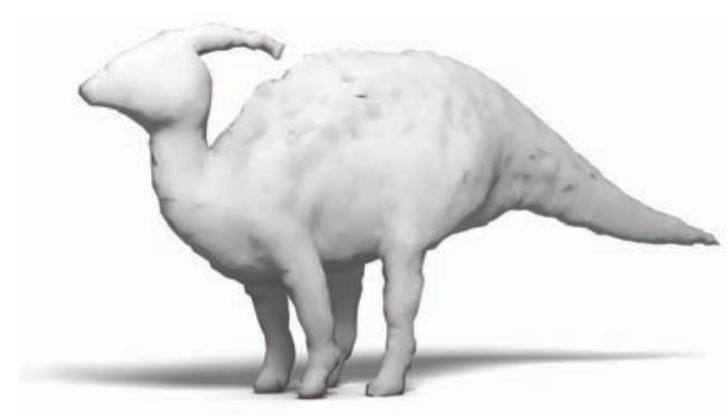

(g) Dinosaur

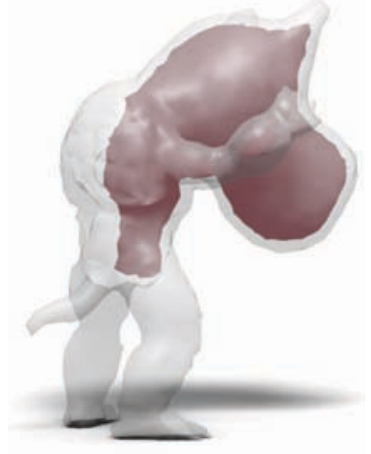

(h) Transparent Armadillo

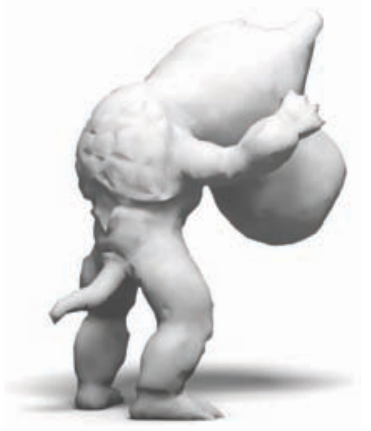

(i) Armadillo

Figure 8: Toy models optimized to improve both stiffness and balance while remaining close to the initial shape.

679 more, a side effect of optimizing a structure to bear its 680 own weight is that the balance is improved.

681 A $10 \mathrm{~cm}$-long plastic model of a dinosaur (source: 682 Aim@Shape) is subjected to external forces (5 MPa) 683 on the tail and the head where one would expect the ${ }_{684}$ model to be weakest. Furthermore, each of the four feet 685 are supported. The SEDs, shape changes and optimized ${ }_{686}$ dinosaur are depicted in Figure 8. Since the external 687 forces are large compared to the gravitational forces, 688 the optimization does not create any cavities. Instead, it 689 redistributes material to places where it improves stiff690 ness. Consequently, compliance is minimized to $46 \%$ 691 of the initial value.

692 Next, a $10 \mathrm{~cm}$-high plastic Armadillo model with 693 a large head (source: Stanford University Computer ${ }_{694}$ Graphics Laboratory and edited in MeshMixer) is sup${ }_{695}$ ported underneath both feet and only subject to gravity. ${ }_{696}$ The SEDs, shape changes and optimized model can be ${ }_{697}$ seen in Figure 8. It is evident that since the model has 698 a large head it will lean forward and thereby subject the 699 shins to large strain. When optimizing compliance, the 700 strain is minimized and the balance of the model is im701 proved as a side effect. However, since imbalance is not 702 directly penalized by the objective function, balance is 703 not guaranteed. A modification of the objective function 704 or constraints would, however, guarantee balance by re705 quiring the center of gravity to stay within the convex
706 hull of the supports.

$$
\text { 707.3. Design }
$$

708 When humans design a given $3 \mathrm{D}$ object, the main 709 concerns are often to satisfy aesthetic and functional 710 requirements. Topology optimization is not concerned 711 with aesthetics but it satisfies functional requirements. 712 However, topology-optimized shapes exhibit an organic 713 and sparse feeling that is often visually pleasing. There714 fore, such a tool is useful as part of a design workflow 715 [47]. Furthermore, the method can be used to generate 716 significantly different designs by slight changes to the 717 input. This is significantly simpler for a designer than 718 remodeling a surface.

719 Three plastic tables are modeled by a fixed layer of 720 material at the top of a design domain $\left(\begin{array}{lll}1.8 & 1.2 & 1.2\end{array}\right.$ $721 \mathrm{~m}^{3}$ ) and a distributed load $(2 \mathrm{MPa})$ pressing down on 722 this layer. Furthermore, three chairs are initialized by 723 filling a $0.6 \quad 0.8 \quad 0.6 \mathrm{~m}^{3}$ design domain. The seat is 724 modeled by a fixed void domain of size $0.4 \quad 0.4 \quad 0.4 \mathrm{~m}^{3}$ 725 and a fixed solid domain underneath which is subjected 726 to a load (1 MPa). Finally, a backrest is modeled by a ${ }_{727}$ small fixed solid domain and subjected to a horizontal 728 force $(0.5 \mathrm{MPa})$. The difference between the problems 729 are the position and extent of the supports. All supports 730 are placed at the bottom of the design domain and have ${ }_{731}$ the shape depicted in figures $9(\mathrm{a}), 9(\mathrm{~d})$ and $9(\mathrm{~g})$ as seen 


\section{ACCEPTED MANUSCRIPT}

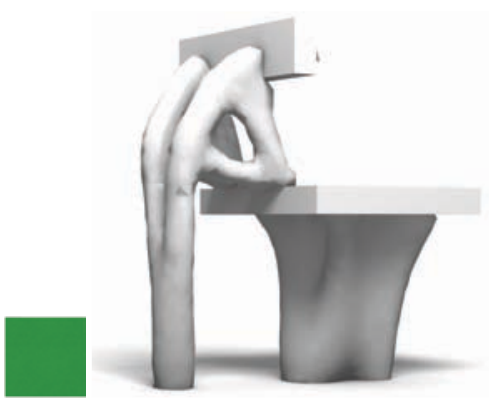

(a)

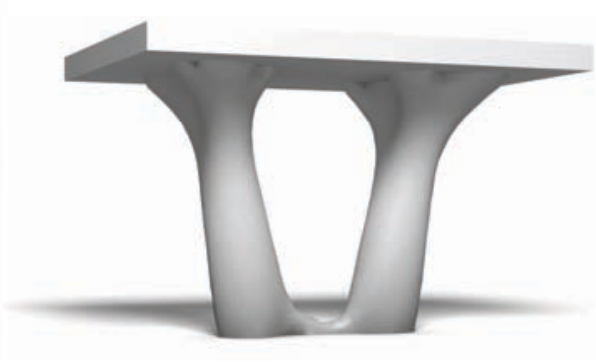

(c) Table 1

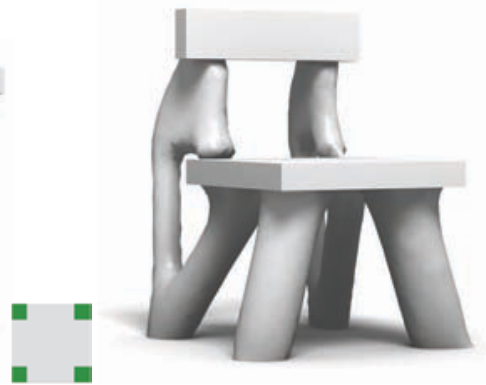

(d) (e) Chair 2

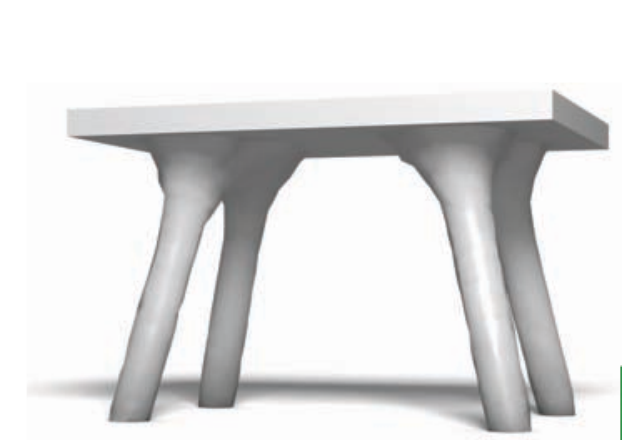

(f) Table 2

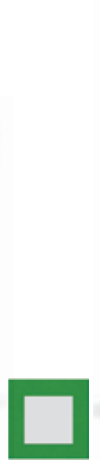

(g)

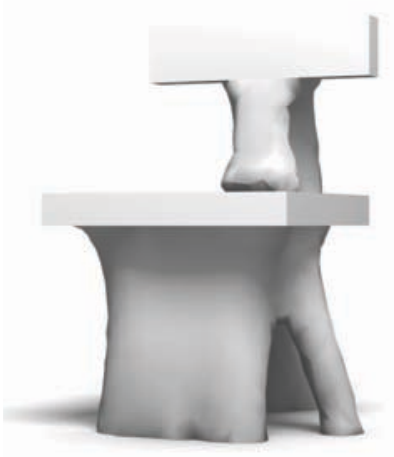

(h) Chair 3

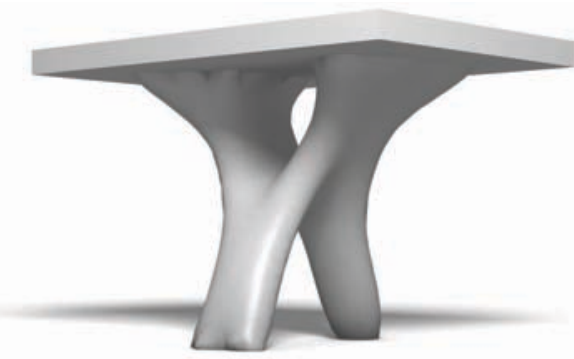

(i) Table 3

Figure 9: Topology optimized tables and chairs which show the design capabilities of the suggested method. The difference between the problems are the supports (illustrated at the left of each row) and possibly the values of parameters. Note that the same illustration is used for both a table and a chair problem, therefore the dimensions of these illustrations are not correct.

732 from above. The optimized designs are depicted in Fig733 ure 9.

${ }_{734}$ Finally, we will use the Qatar National Convention ${ }_{735}$ Center as an example of a real-world architectural de736 sign problem. The Convention Center has an impressive 737 façade which is a roof supported by a concrete topology738 optimized structure [47]. To model this, we take advan739 tage of the symmetry and thereby only optimize a quar740 ter of the structure (the symmetry axes are depicted in ${ }_{741}$ Figure 10(d)). Consequently, the problem is initialized 742 by a $125 \quad 20 \quad 15 \mathrm{~m}^{3}$ cube where the top layer $(1 \mathrm{~m})$ is 743 fixed and solid. The structure is supported at the bottom 744 in a half circular area (Figure 10(d)) and only subjected 745 to gravity. The result can be seen in Figure 10(e) and, in 746 addition, we illustrate in Figure 10 the effect of chang747 ing the parameter for the perimeter constraint. Note that 748 the result is not expected to look like the Convention ${ }_{749}$ Center since [47] use different boundary conditions and 750 do not specify material, objective and constraints.

\section{4. Conclusion}

752 The presented method is the first to optimize both 753 the 3D shape and topology of a surface triangle mesh 754 without the use of an implicit representation. This is 755 achieved by embedding the triangle mesh in a simplicial 756 complex and using the Deformable Simplicial Complex 757 method. Consequently, the method accepts a surface tri758 angle mesh as input and outputs another surface triangle 759 mesh which is only different from the input mesh where 760 it has been optimized. Furthermore, as opposed to stan${ }_{761}$ dard fixed grid methods, our method makes it possible 762 to generate detailed designs within reasonable time on 763 an ordinary laptop.

${ }_{764}$ We have shown that the method automatically gener765 ates designs which satisfy some user-defined structural 766 requirements. However, note that the search space is 767 limited by global constraints and that there is no guaran768 tee that the global optimum is reached. The bridge and 769 the cow statue show that material can be saved where it 770 is expensive or inconvenient while maintaining or im771 proving stiffness. The dinosaur and Armadillo models 772 show that 3D models automatically can be made stiffer 


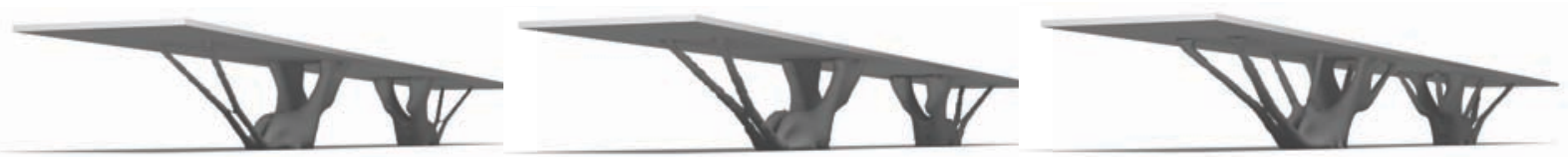

(a) $A^{*}=13 \%$

(b) $A^{*}=15 \%$

(c) $A^{*}=17 \%$

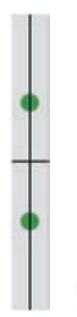

(d)

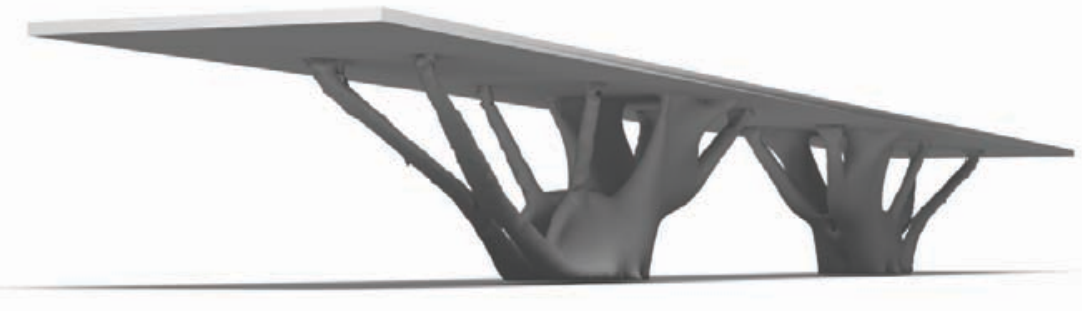

(e) Roof support $\left(A^{*}=20 \%\right)$

Figure 10: Topology optimized roof support, optimized using different values for the perimeter constraint. This problem is inspired by the real world problem of supporting the roof of the Qatar National Convention Center. The supports are placed as depicted in Figure 10(d) where also symmetry axes are visualized as black lines.

773 and more balanced, while retaining the shape. Finally, 774 the tables, chairs and roof support show that functional 775 and, in our opinion, visually pleasing designs can be 776 achieved with little effort from a designer. This is far 777 from an exhaustive list of problems that can be solved 778 using the presented method. As mentioned, topology 779 optimization has been used to solve a wide variety of 780 problems. To solve these or other problems, one only 781 needs to model the boundary conditions and choose the 782 objective, constraints and an initial structure. How783 ever, more advanced problems might require additional 784 work. For example implementing additional objective 785 functions and constraints, handling multiple load cases, 786 using an anisotropic material model, handling dynamic 787 problems and taking non-linearity into account.

${ }_{788}$ We have shown that furniture and support structures 789 for buildings can be modeled by specifying a few in790 put parameters. Furthermore, both the input and output 791 models are in the form of a surface triangle mesh. Con792 sequently, this tool has potential to be used for model793 ing for films, videogames and other offline productions 794 in addition to designing physical structures, especially 795 if performance and user friendliness are improved. To 796 increase performance, one idea is to take full advan797 tage of the parallel nature of the finite element compu798 tations by, for example, feeding the computations to the ${ }_{799}$ GPU. Furthermore, parallelization of the DSC method 800 would be beneficial. Another idea is to take even fur801 ther advantage of the mesh adaptivity by lowering the 802 discretization parameter more wisely. To increase the ${ }_{803}$ user friendliness, automatic determination of worst-case
804 loads could be useful to limit the amount of user input. ${ }_{805}$ Also, finding an alternative to the perimeter constraint 806 would be desirable since it can limit the optimization 807 and its parameter is unintuitive and difficult to choose. 808 Finally, most designers want to influence the design reg809 ularly during the design process. Therefore, a work810 flow which includes user feedback and post processing 811 is needed.

\section{Acknowledgements}

${ }_{813}$ The authors appreciate the support from the Villum ${ }_{814}$ Foundation through the grant: "NextTop"

815 [1] M. P. Bendsøe, N. Kikuchi, Generating optimal topologies in structural design using a homogenization method, Computer Methods in Applied Mechanics and Engineering 71 (2) (1988) $197-224$.

[2] M. P. Bendsøe, O. Sigmund, Topology Optimization - Theory, Methods, and Applications, second edition Edition, Springer Verlag, Berlin, 2003.

[3] M. K. Misztal, J. A. Bærentzen, Topology adaptive interface tracking using the deformable simplicial complex, ACM Transactions on Graphics 31 (3) (2012) No. 24.

[4] H. A. Eschenauer, V. V. Kobelev, A. Schumacher, Bubble method for topology and shape optimization of structures, Structural and Multidisciplinary Optimization 8 (1994) 42-51.

[5] J. Sokolowski, A. Zochowski, On the topological derivative in shape optimization, SIAM Journal on Control and Optimization 37 (4) (1999) 1251-1272.

[6] R. A. Feijóo, A. A. Novotny, E. Taroco, C. Padra, The topological derivative for the poisson's problem, Mathematical Models and Methods in Applied Sciences 13 (12) (2003) 1825-1844.

[7] S. Garreau, P. Guillaume, M. Masmoudi, The topological asymptotic for pde systems: The elasticity case, SIAM J. Control Optim. 39 (6) (2000) 1756-1778. 
[8] F. de Gournay, G. Allaire, F. Jouve, Shape and topology optimization of the robust compliance via the level set method, ESAIM: Control, Optimisation and Calculus of Variations 14 (2008) 43-70

[9] C. Le, T. Bruns, D. Tortorelli, A gradient-based, parameter-free approach to shape optimization, Computer Methods in Applied Mechanics and Engineering 200 (9-12) (2011) 985-996.

10] S. Arnout, M. Firl, K.-U. Bletzinger, Parameter free shape and thickness optimisation considering stress response, Structural and Multidisciplinary Optimization 45 (6) (2012) 801-814.

11] Y. Ding, Shape optimization of structures: a literature survey, Computers \& Structures 24 (6) (1986) 985-1004.

12] B. Mohammadi, F. O. Pironneau, Applied Shape Optimization for Fluids, Oxford University Press, 2009.

13] D. Bucur, G. Buttazzo, Variational Methods in Shape Optimization Problems, Progress in Nonlinear Differential Equations and Their Applications, Birkhäuser, 2006.

14] K. Svanberg, The method of moving asymptotes - a new method for structural optimization, International Journal for Numerical Methods in Engineering 24 (2) (1987) 359-373.

5] R. Prévost, E. Whiting, S. Lefebvre, O. Sorkine-Hornung, Make It Stand: Balancing shapes for 3D fabrication, ACM Transactions on Graphics (proceedings of ACM SIGGRAPH) 32 (4) (2013) 81:1-81:10.

6] M. Skouras, B. Thomaszewski, S. Coros, B. Bickel, M. Gross, Computational design of actuated deformable characters, ACM Trans. Graph. 32 (4) (2013) 82:1-82:10.

7] F. De Goes, P. Alliez, H. Owhadi, M. Desbrun, On the Equilibrium of Simplicial Masonry Structures, ACM Transactions on Graphics 32 (4).

18] Y. Liu, H. Pan, J. Snyder, W. Wang, B. Guo, Computing self-supporting surfaces by regular triangulation, ACM Trans. Graph. 32 (4) (2013) 92:1-92:10.

19] D. Panozzo, P. Block, O. Sorkine-Hornung, Designing unreinforced masonry models, ACM Transactions on Graphics (proceedings of ACM SIGGRAPH) 32 (4) (2013) 91:1-91:12.

20] N. Umetani, R. Schmidt, Cross-sectional structural analysis for 3d printing optimization, in: SIGGRAPH Asia 2013 Technical Briefs, SA '13, ACM, New York, NY, USA, 2013, pp. 5:1-5:04.

21] O. Stava, J. Vanek, B. Benes, N. Carr, R. Mech, Stress relief: Improving structural strength of $3 \mathrm{~d}$ printable objects, ACM Trans. Graph. 31 (4) (2012) 48:1-48:11.

2] Q. Zhou, J. Panetta, D. Zorin, Worst-case structural analysis, ACM Trans. Graph. 32 (4) (2013) 137:1-137:12

M. P. Bendsøe, Optimal shape design as a material distribution problem, Structural Optimization 1 (4) (1989) 193-202. plicit Surfaces, 1st Edition, Springer, 2002

5] M. Wang, X. Wang, D. Guo, A level set method for structural topology optimization, Computer Methods in Applied Mechanics and Engineering 192 (1) (2003) 227-246.

26] G. Allaire, F. Jouve, A.-M. Toader, Structural optimization using sensitivity analysis and a level-set method, Journal of Computational Physics 194 (1) (2004) 363-393.

7] S.-H. Ha, S. Cho, Level set based topological shape optimization of geometrically nonlinear structures using unstructured mesh, Computers \& Structures 86 (13-14) (2008) 1447-1455.

28] G. Allaire, C. Dapogny, P. Frey, Topology and geometry optimization of elastic structures by exact deformation of simplicial mesh, Comptes Rendus Mathematique 349 (17-18) (2011) 999_ 1003.

9] S. Yamasaki, T. Nomura, A. Kawamoto, K. Sato, S. Nishiwaki, A level set-based topology optimization method targeting metallic waveguide design problems, International Journal for $\mathrm{Nu}$ merical Methods in Engineering 87 (9) (2011) 844-868.
902 [30] Q. Xia, T. Shi, S. Liu, M. Y. Wang, A level set solution to the stress-based structural shape and topology optimization, Computers \& Structures 90 - 91 (0) (2012) 55-64.

05 [31] G. Allaire, C. Dapogny, P. Frey, A mesh evolution algorithm based on the level set method for geometry and topology optimization, Structural and Multidisciplinary Optimization 48 (4) (2013) 711-715.

909 [32] K. Maute, E. Ramm, Adaptive topology optimization, Structural optimization 10 (1995) 100-112.

911 [33] A. N. Christiansen, M. Nobel-Jørgensen, N. Aage, O. Sigmund, J. A. Bærentzen, Topology optimization using an explicit interface representation, Structural and Multidisciplinary Optimization 49 (3) (2014) 387-399.

15 [34] H. Si, TetGen: A quality tetrahedral mesh generator and a 3d delaunay triangulator (2013). URL http://wias-berlin.de/software/tetgen/

18 [35] V. N. Parthasarathy, C. M. Graichen, A. F. Hathaway, A comparison of tetrahedron quality measures, Finite Elem. Anal. Des. 15 (3) (1994) 255-261.

1 [36] D. A. Field, Laplacian smoothing and delaunay triangulations, Communications in Applied Numerical Methods 4 (6) (1988) 709-712.

24 [37] J. A. Bærentzen, J. Gravesen, F. Anton, H. Aanæs, Guide to Computational Geometry Processing: Foundations, Algorithms, and Methods, Springer, 2012.

27 [38] J. R. Shewchuk, Two discrete optimization algorithms for the topological improvement of tetrahedral meshes, in: Unpublished manuscript, 2002.

30 [39] R. D. Cook, D. S. Malkus, M. E. Plesha, R. J. Witt, Concepts and Applications of Finite Element Analysis, John Wiley \& Sons, 2007.

[40] Y. Chen, T. A. Davis, W. W. Hager, S. Rajamanickam, Algorithm 887: Cholmod, supernodal sparse cholesky factorization and update/downdate, ACM Transactions on Mathematical Software 35 (3) (2008) 22:1-22:14.

[41] T. A. Davis, W. W. Hager, I. S. Duff, SuiteSparse (2013). URL http://www.cise.ufl.edu/research/sparse/ SuiteSparse/

40 [42] R. Haber, C. Jog, M. Bendsøe, A new approach to variabletopology shape design using a constraint on perimeter, Structural optimization 11 (1-2) (1996) 1-12.

43 [43] O. Pironneau, Optimal shape design for elliptic systems, in: R. Drenick, F. Kozin (Eds.), System Modeling and Optimization, Vol. 38 of Lecture Notes in Control and Information Sciences, Springer Berlin Heidelberg, 1982, pp. 42-66.

47 [44] P. W. Christensen, A. Klarbring, An Introduction to Structural Optimization, Solid mechanics and its applications, Springer, 2008.

50 [45] J. Céa, S. Garreau, P. Guillaume, M. Masmoudi, The shape and topological optimizations connection, Computer Methods in Applied Mechanics and Engineering 188 (4) (2000) 713-726.

53 [46] A. N. Christiansen, R. Schmidt, J. A. Bærentzen, Automatic balancing of 3d models, Computer-Aided Design (2014) to appear.

955 [47] M. Sasaki, T. Itō, A. Isozaki, Morphogenesis of flux structure, AA Publications, 2007. 